\title{
Fluid-structure interaction model of human mitral valve within left ventricle
}

\author{
Hao Gao ${ }^{1}$, Nan $\mathrm{Qi}^{1}$, Ma Xingshuang ${ }^{2}$, Boyce E. Griffith ${ }^{3}$, Colin Berry ${ }^{4}$, and \\ Xiaoyu Luo $^{1}$ \\ ${ }^{1}$ School of Mathematics and Statistics, University of Glasgow, UK \\ ${ }^{2}$ School of Aerospace Engineering, Chongqing University, China \\ 3 Department of Mathematics, University of North Carolina, Chapel Hill, NC, USA \\ ${ }^{4}$ Institute of Cardiovascular and Medical Science, University of Glasgow, UK
}

\begin{abstract}
We present an integrated model of mitral valve coupled with the left ventricle. The model is derived from clinical images and takes into account of the important valvular features, left ventricle contraction, nonlinear soft tissue mechanics, fluid structure interaction, and the MVLV interaction. This model is compared with a corresponding mitral-tube model, and differences in the results are discussed. Although the model is a step closer towards simulating physiological realistic situation, further work is required to ensure that the highly complex valvular-ventricular interaction, and the fluid-structure interaction, can be reliably represented.
\end{abstract}

\section{Introduction}

Moderate or severe mitral valve (MV) dysfunction remains a major medical problem. It can be caused, among others, by leaflet prolapse or is secondary to left ventricular diseases [15]. Computational modelling of the MV mechanics, particularly within the context of the left ventricle (LV) environment, can enhance our understanding of the valvular-ventricular interaction, and potentially lead to more efficient MV repairs and replacement.

Research on developing biomechanical MV models can be dated back to 1990s [10]. Recent development involves finite strain deformational kinematics, realistic anatomical geometries and advanced hyperelastic constitutive models [2, $16,17]$. However, most of the studies are based on purely structural analysis with applications to a statically or dynamically pressurized closed valve in isolated situation [9]. Since the structure of the MV is closely tied to the left ventricle through the chordae connection, it is important to simulating the dynamics of MV by taking into account of LV dynamics, as well as the fluid-structure interaction (FSI) between the MV, LV and the blood.

Kunzelman, Einstein and co-workers first started to simulate normal and pathological mitral function $[3,4,11]$ with FSI. Over the last few years there have been a number of FSI valvular models using the immersed boundary (IB) approach $[6,7,13,18]$. However, none of these MV models included the effect of the LV motion, hence the flow field is not physiological. Indeed, Lau et al [12] 
compared the MV dynamics in a straight tube and a U-shaped ventricle, and found that when the MV is mounted into a LV, the transvalvular velocity is slower compared to the one estimated using a tubular geometry. Yin et al. [19] modelled a chordaed MV inside a LV and identified fluid vortices associated with the LV motion. However, their LV motion is modelled as a set of prescribed moving boundary, and the MV model is simply constructed using a network of linear elastic fibres. Chandran and Kim [1] recently reported a prototype FSI MV dynamics in a simplified LV chamber model using an immersed interface-like approach. To date, there has been no work reported that included both the MV and LV models and the fluid-structure interaction properly.

In this study, we have developed a fully integrated MV-LV model, which is image-derived and simulated using a hybrid immersed boundary-finite element framework (IB/FE) [8]. We will also compare the differences of the MV dynamics when the MV is mounted in the $\mathrm{LV}$ and in a tube.

\section{Methodology}

The hybrid IB/FE method employs an Eulerian description of the viscous incompressible fluid, described by $\mathbf{x}=\left(x_{1}, x_{2}, x_{3}\right) \in \Omega$, along with a Lagrangian description of the structure that is immersed in the fluid, described by $\mathbf{X}=$ $\left(X_{1}, X_{2}, X_{3}\right) \in U$. Interactions between the Lagrangian $(\mathbf{X})$ and Eulerian $(\mathbf{x})$ fields are achieved by integral transforms with a Dirac delta function kernel $\delta(\mathbf{x})$. In brief, the IB form of the equations of motion is:

$$
\begin{aligned}
\rho\left(\frac{\partial \mathbf{u}}{\partial t}(\mathbf{x}, t)+\mathbf{u}(\mathbf{x}, t) \cdot \nabla \mathbf{u}(\mathbf{x}, t)\right) & =-\nabla p(\mathbf{x}, t)+\mu \nabla^{2} \mathbf{u}(\mathbf{x}, t)+\mathbf{f}^{\mathrm{s}}(\mathbf{x}, t) \\
\nabla \cdot \mathbf{u}(\mathbf{x}, t) & =0 \\
\mathbf{f}^{\mathrm{s}}(\mathbf{x}, t) & =\int_{U} \nabla \cdot \mathbb{P}^{\mathrm{s}}(\mathbf{X}, t) \delta(\mathbf{x}-\boldsymbol{\chi}(\mathbf{X}, t)) \mathrm{d} \mathbf{X} \\
& -\int_{\partial U} \mathbb{P}^{\mathrm{s}}(\mathbf{X}, t) \mathbf{N}(\mathbf{X}) \delta(\mathbf{x}-\boldsymbol{\chi}(\mathbf{X}, t)) \mathrm{d} A(\mathbf{X}), \\
\frac{\partial \boldsymbol{\chi}}{\partial t}(\mathbf{X}, t) & =\int_{\Omega} \mathbf{u}(\mathbf{x}, t) \delta(\mathbf{x}-\boldsymbol{\chi}(\mathbf{X}, t)) \mathrm{d} \mathbf{x}
\end{aligned}
$$

in which $\rho$ is the mass density, $\mu$ is the viscosity, $\mathbf{u}(\mathbf{x}, t)$ is the Eulerian velocity filed of the system, $p(\mathbf{x}, t)$ is the Eulerian pressure field, $\mathbf{f}^{s}(\mathbf{x}, t)$ is the Eulerian elastic force density. $\boldsymbol{\chi}(\mathbf{X}, t) \in \Omega$ denotes the physical position of material point $\mathbf{X}$ at time $t$, and $\mathbf{N}(\mathbf{X})$ is the exterior unit normal at $\partial U . \mathbb{P}^{\mathrm{s}}$ is the first PiolaKirchoff stress tensor, which is related to the Cauchy stress $\sigma^{\mathrm{s}}$ of the immersed structure by $\mathbb{P}^{\mathrm{s}}=\operatorname{det}(\mathbb{F}) \sigma^{\mathrm{s}} \mathbb{F}^{-T}$, in which $\mathbb{F}=\partial \boldsymbol{\chi} / \partial \mathbf{X}$ is the deformation gradient. $\mathbb{P}^{\mathrm{s}}$ is determined from the material properties by strain energy functional $W$ by $\mathbb{P}^{s}=\partial W / \partial \mathbb{F}$. Readers may refer to [8] for more details of the IB/FE method.

A cardiac magnetic resonance (CMR) study was performed on a healthy young volunteer. The study was approved by the local NHS Research Ethics 
(a)

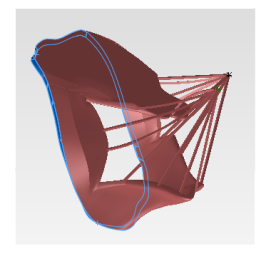

(b)

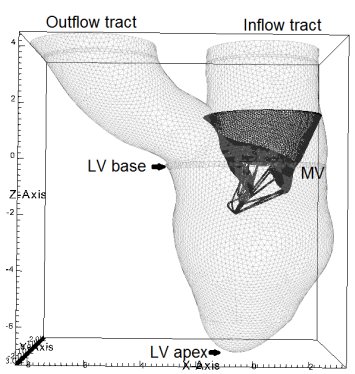

(c)

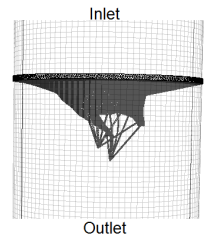

Fig. 1: The MRI-derived MV model (a), the MRI-derived MV-LV model (b), and the MV-tube model (c).

Committee, and written informed consent was obtained before the CMR scan. Twelve imaging planes along the LV outflow tract view were imaged to cover the MV with cine images. Typical parameters were: slice thickness: $3 \mathrm{~mm}$; inplane pixel size: $0.7 \times 0.7 \mathrm{~mm}^{2}$, field of view: $302 \times 400 \mathrm{~mm}^{2}$; frame rate: 25 per cardiac cycle. The MV geometry was reconstructed from a stack of cine images at early-diastole when it first opens, as shown in Fig. 1(a), with sixteen chordae distributed evenly and running through the leaflet free edges to the annulus ring. Details on the subject-specific MV reconstruction from CMR images are similar as in $[6,13]$. Short-axial and long-axial cine images were also performed to cover the LV region. Typical parameters were: slice thickness: $10 \mathrm{~mm}$; in-plane pixel size: $1.3 \times 1.3 \mathrm{~mm}^{2}$; frame rate: 25 per cardiac cycle. The LV geometry including the inflow and outflow tracts was reconstructed from the same volunteer at early of diastole just before the MV opens (Fig. 1(b)) [5]. The MV geometry is mounted to the inflow tract according to the relative positions derived from the CMR images. This forms the integrated MV-LV model. Following the work in [6], the MV is also fixed to a housing disc and mounted to a rigid outer tube (Fig. 1(c)), this forms the MV-tube model.

Simulations of the MV-LV and MV-tube models are run using the opensource IBAMR software framework ${ }^{5}$, which provides an adaptive and distributedmemory parallel infrastructure for developing the IB/FE models. In the MV-LV model, the structure below LV base is contractile, the regions above the LV, including the MV and its apparatuses, are made to only bear the load passively. The LV base is allowed to have radial expansion, but fixed in the long axial and circumferential directions. During diastole, zero flow boundary condition is imposed in the outflow tract. The pressure that is linearly ramped to the enddiastolic pressure (assumed to be $8 \mathrm{mmHg}$ ) is applied in the inflow tract in $0.8 \mathrm{~s}$. After the end-diastole, the LV region simultaneously contracts, triggered by a spatially homogeneous intracellular calcium transient [5].

\footnotetext{
${ }^{5}$ https://ibamr.googlecode.com
} 
The increased LV pressure will close the MV and open the aortic valve when the LV pressure exceeds the diastolic aortic pressure, assumed to be the measured diastolic cuff pressure from the healthy volunteer $(85 \mathrm{mmHg})$. Because the aortic valve is not included in the MV-LV model, the aortic tract is either fully open or fully closed, determined by the pressure difference between the value inside LV and the aorta. During the systolic ejection, a three-element Windkessel model [7] is connected to the outflow tract to provide a physiological pressureflow boundary condition, the systolic phase ends when the LV no longer pumps blood out. The end-diastolic pressure $(8 \mathrm{mmHg})$ is maintained in the inflow boundary until the end-systole. The chordae are not directly attached to the LV wall, but modelled similarly as in [6]. In the MV-tube model, typical pressure profiles are applied on the inlet and outlet boundaries, as described in [6].

\section{$3 \quad$ Results}

Because of the increased pressure in the inflow tract during diastole, the volumetric flow rate across the MV linearly increases until end of diastole, with a maximum value of $90 \mathrm{~mL} / \mathrm{s}$. The total inflow volume across the MV in diastole is $40 \mathrm{~mL}$, which is less than the real cardiac output (around $80 \mathrm{~mL}$ ). Fig.2(a) shows the deformed MV leaflets of the MV-LV model when the MV is fully open. This is compared to that of the MV-tube model (Fig.2(b)). We can see that the orifice in the MV-LV model is smaller, which suggests that the LV wall provides more resistance to the blood flow that fills the LV cavity during the MV opening. This does not happen in the MV-tube model, hence for the same pressure drop, the flow rate is greater. In systole, it takes 80 milliseconds for the LV model to develop high enought pressure in order to eject the blood through the outflow tract. The closing regurgitation flow across the MV is estimated to be $7.2 \mathrm{~mL}$.

Fig. 2(c) shows the deformed MV leaflets when the LV starts to eject blood. Notice that the MV in the MV-LV model is only partially closed, compared to that of the MV-tube model in Fig. 2(d). The MV regurgitation in the MV-LV model persists in systole, which prevents the ventricular pressure to increase efficiently. The peak value of the pressure is $117 \mathrm{mmHg}$, which is much less than the peak value of $160 \mathrm{mmHg}$, corresponding to no leak at the inflow tract in systole.

Figs. 3 (a, b, c, d) show, respectively, the streamlines in the MV-LV model during the early diastolic filling, the late diastolic filling, when the MV is closing, and when the LV is ejecting blood through the outflow tract. The corresponding streamlines of the MV-tube model is shown in Figs. 3 (e, f, g, h). In the MV-LV model, the flow moves directly towards the LV apex in the early filling, forming large vortices in the late diastolic filling in the whole LV cavity, mixing the fresh blood from left atrium with the remaining blood from the previous heart beat. These flow features are absent in the MV-tube model. 
(a)

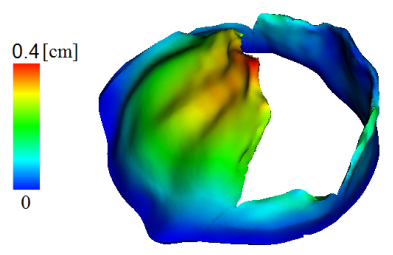

(c)

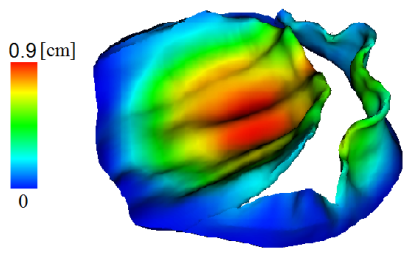

(b)

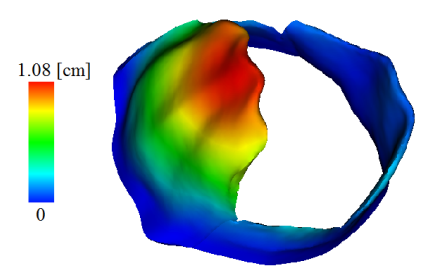

(d)

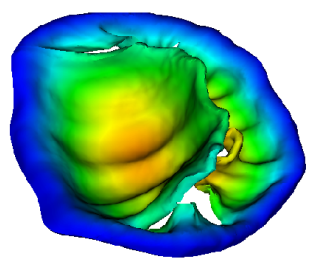

Fig. 2: The fully opened MV in (a) the MV-LV model, (b) the MV-tube model (b). The closed MV in (c) the MV-LV model, and (d) the MV-tube model (d). Coloured by the displacement.

\section{Discussion and Conclusion}

In this study, we have built an integrated MV-LV model based on in vivo CMR images of a healthy volunteer. This model incorporates a MV, a contractile LV, and the fluid structure interaction for the first time. Results are compared with that of a corresponding MV-tube model, and a number of differences are noticed. First, the flow patterns are very different in the MV-LV model. When the MV is opened, the blood passes through the MV and is directed to the posterior side in the MV-tube model before hitting the tubular wall. However, in the MV-LV model, the flow goes directly towards the LV apex, and then bends toward LV base to form large vortices. The MV-tube model is unable to model the flow in systole due to the absence of the aortic part. Those differences suggest that the incorporation of the left ventricle (the valvular-ventricle interaction) is necessary for modelling the flow patterns around the MV. However, if one is only interested in the MV closure, then a reduced-model (MV only) may be sufficient [6, 17].

During diastole, the MV-LV model seems to produce a smaller orifice compared to the corresponding MV-tube model. This is because of the extra resistance offered by the LV wall, which is absent in the MV-tube model. Consequently, the total inflow volume through the MV is reduced. According to [14], the diastolic phase could be divided into three phases as the rapid filling, slow filling and atrial contraction. In the rapid filling, the transvalvular flow is resulted from the relaxation of the LV (the sucking effect), and $80 \%$ transvalvular flow occurs [14]. During the slow filling and atrial contraction, the left atrium needs to generate higher pressure for further filling. In the MV-LV model, the 
ramped pressure in the inflow tract during diastole is similar to the slow filling and atrial contraction. However, the model is less accurate in simulating the rapid filling phase. This suggest that our boundary conditions need to be improved. We also notice that during systole, the MV does not close as well as in the MV-tube model, suggesting that the deformation of the annulus ring and the papillary muscles may have to be included in order to produce a physiological fluid mechanics around the MV.

The heart function is well coordinated through the electrical-mechanical coupling, the valvular closing and opening events, etc., thus the combination of the MV and LV models is more challenging compared to the isolated MV or LV models. Furthermore due to the large number of parameters involved in the integrated MV-LV model, we have not achieved a fully personalized MV-LV model (much less stroke volume compared to the measurements), though the MV and LV models are from the same volunteer. Currently we are working towards optimizing model parameters, estimating pressure boundaries, and incorporating more detailed valvular-ventricular interactions.

\section{Acknowledgement}

This work is funded by the UK EPSRC (EP/I1029990), and the British Heart Foundation (PG/14/64/31043, PG/11/2/28474). B.E.G. acknowledges research support from the American Heart Association (AHA award 10 SDG4320049), the National Institutes of Health (award HL117063), and the National Science Foundation (awards DMS 1016554 and ACI 1047734).

\section{References}

1. Chandran, K.B., Kim, H.: Computational mitral valve evaluation and potential clinical applications. Annals of biomedical engineering pp. 1-15 (2014)

2. Conti, C.A., Stevanella, M., Maffessanti, F., Trunfio, S., Votta, E., Roghi, A., Parodi, O., Caiani, E.G., Redaelli, A.: Mitral valve modelling in ischemic patients: Finite element analysis from cardiac magnetic resonance imaging. In: Computing in Cardiology, 2010. pp. 1059-1062. IEEE (2010)

3. Einstein, D.R., Kunzelman, K.S., Reinhall, P.G., Nicosia, M.A., Cochran, R.P.: Non-linear fluid-coupled computational model of the mitral valve. Journal of Heart Valve Disease 14(3), 376-385 (2005)

4. Einstein, D.R., Reinhall, P., Nicosia, M., Cochran, R.P., Kunzelman, K.: Dynamic finite element implementation of nonlinear, anisotropic hyperelastic biological membranes. Computer Methods in Biomechanics and Biomedical Engineering 6(1), 33-44 (2003)

5. Gao, H., Carrick, D., Berry, C., Griffith, B.E., Luo, X.: Dynamic finite-strain modelling of the human left ventricle in health and disease using an immersed boundary-finite element method. IMA Journal of Applied Mathematics (2014)

6. Gao, H., Ma, X., Qi, N., Berry, C., Griffith, B.E., Luo, X.: A finite strain nonlinear human mitral valve model with fluid-structure interaction. International journal for numerical methods in biomedical engineering 30(12), 1597-1613 (2014) 
7. Griffith, B.E.: Immersed boundary model of aortic heart valve dynamics with physiological driving and loading conditions. International Journal for Numerical Methods in Biomedical Engineering 28(3), 317-345 (2012)

8. Griffith, B.E., Luo, X.: Hybrid finite difference/finite element version of the immersed boundary method. Submitted, preprint available from http://www. cims. nyu. edu/ griffith (2012)

9. Kamensky, D., Hsu, M.C., Schillinger, D., Evans, J.A., Aggarwal, A., Bazilevs, Y., Sacks, M.S., Hughes, T.J.: An immersogeometric variational framework for fluid-structure interaction: Application to bioprosthetic heart valves. Computer methods in applied mechanics and engineering (2014)

10. Kunzelman, K.S., Cochran, R.: Stress/strain characteristics of porcine mitral valve tissue: parallel versus perpendicular collagen orientation. Journal of cardiac surgery $7(1), 71-78(1992)$

11. Kunzelman, K.S., Einstein, D.R., Cochran, R.P.: Fluid--structure interaction models of the mitral valve: function in normal and pathological states. Philosophical Transactions of the Royal Society B: Biological Sciences 362(1484), 1393-1406 (2007)

12. Lau, K., Diaz, V., Scambler, P., Burriesci, G.: Mitral valve dynamics in structural and fluid-structure interaction models. Medical engineering \& physics 32(9), 10571064 (2010)

13. Ma, X., Gao, H., Griffith, B.E., Berry, C., Luo, X.: Image-based fluid-structure interaction model of the human mitral valve. Computers \& Fluids 71, 417-425 (2013)

14. Nishimura, R.A., Tajik, A.J.: Evaluation of diastolic filling of left ventricle in health and disease: Doppler echocardiography is the clinician?s rosetta stone. Journal of the American College of Cardiology 30(1), 8-18 (1997)

15. Ray, R., Chambers, J.: Mitral valve disease. International journal of clinical practice (2014)

16. Sun, W., Abad, A., Sacks, M.S.: Simulated bioprosthetic heart valve deformation under quasi-static loading. Journal of biomechanical engineering 127(6), 905-914 (2005)

17. Wang, Q., Sun, W.: Finite element modeling of mitral valve dynamic deformation using patient-specific multi-slices computed tomography scans. Annals of biomedical engineering 41(1), 142-153 (2013)

18. Watton, P.N., Luo, X.Y., Yin, M., Bernacca, G.M., Wheatley, D.J.: Effect of ventricle motion on the dynamic behaviour of chorded mitral valves. Journal of Fluids and Structures 24(1), 58-74 (2008)

19. Yin, M., Luo, X., Wang, T., Watton, P.: Effects of flow vortex on a chorded mitral valve in the left ventricle. International Journal for Numerical Methods in Biomedical Engineering 26(3-4), 381-404 (2010) 
(a)

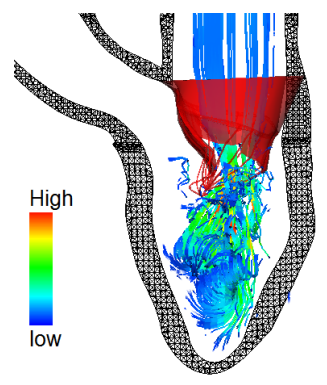

(c)

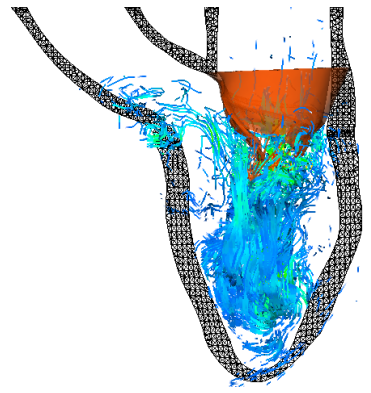

(e)

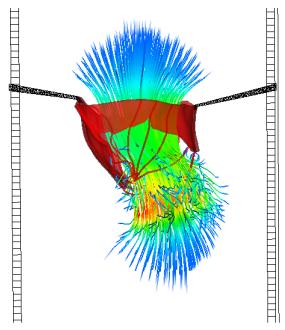

(g)

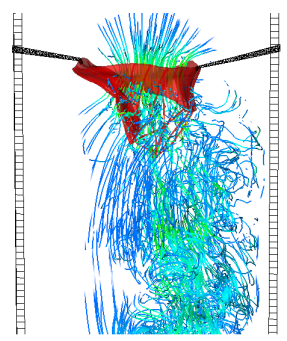

(b)

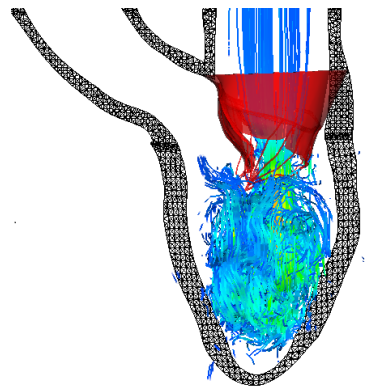

(d)

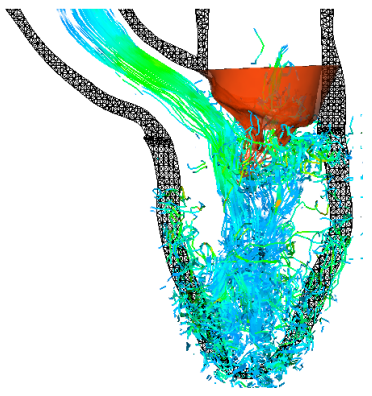

(f)

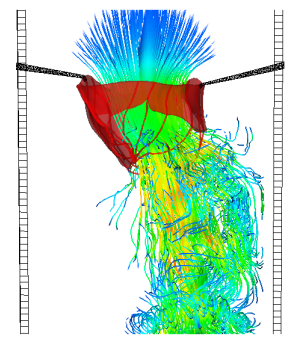

(h)

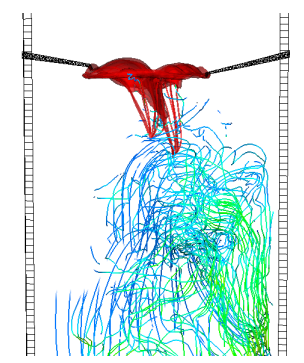

Fig. 3: Streamlines in the MV-LV model at the early diastolic filling (a), at the late diastolic filling (b), when the MV is closing (c), and at the middle of the systolic ejection (d). Streamlines in the MV-tube model at the early diastolic filling (e), at the late diastolic filling (f), when the MV is closing (g) and when the MV is fully closed (h). Coloured by the velocity magnitude. 